\title{
Adaptación y evaluación de las propiedades psicométricas del brief-p en preescolares colombianos
}

\section{Adaptation and evaluation of the psychometric properties of brief-p in colombian preschoolers}

\author{
Maritza Alexandra Rincón Díaz ${ }^{1}$ - César Armando Rey Anacona 2 ( \\ 'Universidad de San Buenaventura Sede Bogotá \\ Colombia
}

Fecha correspondencia:

Recibido: marzo 18 de 2016.

Aceptado: diciembre 16 de 2016.

Forma de citar:

Rincón, M. \&Rey, C. A., (2017).

Adaptación y evaluación de

las propiedades psicométricas

del Brief-P en preescolares

colombianos. Rev. CES Psicol.,

10(1), 48-62.

Open access

(c) Copyright

Licencia creative commons

Etica de publicaciones

Revisión por pares

Gestión por Open Journal System

DOl: http://dx.doi.org/10.21615/

cesp.10.1.4

ISSN: 2011-3080

Sobre los autores:

1. Magister en Neuropsicología

Clínica, Especialista en Familia,

Docente Institución Educativa

Panamericano Puente Boyacá.

2. Doctor en Psicología Clínica y de la Salud, Docente-investigador

Comparte

\section{Resumen}

Las funciones ejecutivas (FE) son una serie de procesos que gestionan y coordinan las demás funciones cognitivas, las respuestas emocionales y comportamentales, especialmente ante problemas novedosos. Su evaluación es importante en los años preescolares con el fin de prevenir problemas de aprendizaje y de comportamiento. En este estudio se adaptó el Behavior Rating Inventory of Executive Function - Preschool Version (BRIEF-P) y se evaluaron sus propiedades psicométricas con una muestra de 125 niños(as) colombianos entre 2 y 4 años y once meses de edad, mediante la implementación de diversas pruebas de confiabilidad y validez. Se encontraron índices altos de estabilidad test-retest y de consistencia interna, en el puntaje global, los tres índices y las cinco escalas del instrumento, y una estructura de correlaciones superiores a .50 entre dichas escalas. Cinco factores explicaban el $100 \%$ de la varianza y las escalas del BRIEF-P correlacionaron significativamente con el puntaje obtenido en pruebas de desempeño de FE diseñadas para preescolares. Estos datos confirman las adecuadas propiedades psicométricas del instrumento, aunque se recomienda un estudio de baremación.

Palabras clave: Funciones Ejecutivas, Evaluación, Preescolares, BRIEF-P, Validez, Confiabilidad.

\section{Abstract}

Executive functions are a series of processes that regulate and coordinate cognitive functions, emotional and behavioral responses, and the ability to deal with novelty. Evaluating executive functions in preschool years may prevent learning and behavior problems. In this study, It was adapted the Behavior Rating Inventory of Executive Function - Preschool Version (BRIEF-P), and its psychometric properties were evaluated sampling 125 Colombian children between 2 and 4 years and eleven months old, through the implementation of several tests of reliability and validity. It was found high rates of test-retest reliability and internal consistency in the overall score, the three rates and the five scales of the instrument, and a structure of correlations above .50 among these scales. Five factors accounted for $100 \%$ of the variance and the BRIEF-P scales correlated significantly with 
Pág 49

Universidad Pedagógica y

Tecnológica de Colombia - UPTC

- Sede Tunja y Universidad de

San Buenaventura Sede Bogotá,

Colombia. the achieved score in performance tests designed for evaluating executive functions in preschoolers. These data confirm the adequate psychometric properties of the instrument, although a scale study is recommended.

Keywords: Executive Functions, Evaluation, Preschool, BRIEF-P, Test Validity, Test Reliability.

\section{Introducción}

Las funciones ejecutivas (FE) son aquellos procesos encargados de gestionar y coordinar las funciones cognitivas, las respuestas emocionales y comportamentales, especialmente ante problemas novedosos (Goia, Espy, \& Isquith, 2003), e incluyen funciones como la memoria de trabajo, la inhibición de respuestas inapropiadas y la monitorización de la conducta con respecto a estados motivacionales y emocionales del organismo, teniendo como principal sustrato neuro-anatómico la corteza prrefrontal y sus conexiones recíprocas con otras zonas del córtex cerebral y varias estructuras subcorticales (Isquith, Crawford, Espy, \& Gioia, 2005; Samango-Sprouse, 2007; Tirapu \& Luna-Lario, 2008; Trujillo \& Pineda, 2008; Zelazo, 2003).

El desarrollo de las FE es determinante en algunos períodos del desarrollo como la primera infancia, en el que se espera que se sienten las bases para un óptimo desarrollo en las esferas cognitiva, social y emocional (Banco Mundial, 2011; National Scientific Council on the Developing Child, 2007; Oates, Karmiloff-Smith, \& Johnson, 2012; Walker et al., 2011). Sin embargo, dicho desarrollo puede alterarse por factores pre y peri natales como anormalidades genéticas, prematuridad, la exposición a tóxicos en el embarazo y traumas cráne-encefálicos en los primeros años de vida, predisponiendo al desarrollo de trastornos del aprendizaje y del comportamiento (Azcoaga, 2008; Espy, Kaufmann, Glisky, \& McDiarmid, 2001; Flores, Ostroksy-Solís, \& Lozano, 2008; Isquith et al., 2005; Pérez \& Capilla, 2008; Stelzer, Cervigni, \& Martino, 2011; Thorella \& Wåhlstedt, 2006). Por lo tanto, la medición de las FE a temprana edad resulta indispensable para detectar posibles déficits e implementar acciones que permitan corregirlos y prevenir de esta manera diversas dificultades.

Sin embargo, el número de instrumentos que existen para evaluar las FE en preescolares es escaso y su desarrollo se ha basado fundamentalmente en la adaptación de pruebas para adultos y en la evaluación de menores con disfunción del lóbulo frontal causada por diversas patologías, más que en el desarrollo normal de estas funciones durante esta etapa del desarrollo (Carlson, 2005; Fletcher, 1996; González \& Ostrosky, 2012; Isquith et al., 2005). Además, los efectos de las lesiones en la corteza sobre las FE son diferentes en los niños(as) con respecto a los adultos, debido al desarrollo progresivo de estas funciones, la mielinización tardía de las estructuras involucradas y la plasticidad cerebral (Sánchez-Carpintero \& Narbona, 2004).

Si bien existe consenso en el sentido de que las FE no aparecen antes de los dos años de edad, en el primer año de vida ocurre una serie de cambios anatómicos y funcionales que inciden en el funcionamiento de la corteza prefrontal y sus conexiones, que pemiten el desarrollo de las habilidades necesarias para el surgimiento de las FE, como la permanencia de objeto, que se manifiesta hacia los ocho meses de edad y se considera una habilidad precursora de la memoria de trabajo (Carlson, 2005; Roselli, Jurado, \& Matute, 2008; Stelzer et al., 2011). Hacia los cuatro años se da un cambio significativo en la capacidad de anticipar dificultades en la resolución de problemas sencillos, en la planificación y en la inhibición de respuestas habituales, facilitando el surgimiento de la teoría de la mente y el incremento de la regulación emocional (Pérez \& Capilla, 2008). 
Pág 50

El BRIEF-P no ha sido todavía validado en países como Colombia, donde se requiere este tipo de instrumentos de evaluación, debido a la presencia de condiciones adversas para el sano desarrollo de los niños(as), como la desigualdad, el conflicto armado, los malos tratos infantiles y el consumo abusivo de bebidas alcohólicas, así como la alta prevalencia de trastornos como el TDAH (Ministerio de Salud de Colombia \& Colciencias, 2015), asociados a déficits en $\mathrm{FE}$ que requieren una evaluación a temprana edad.
En los años preescolares también emerge la capacidad de utilizar sistemas jerárquicos de representaciones mentales para regular el comportamiento conforme a reglas, favoreciendo la adquisición de habilidades sociales y la regulación de las emociones (Carlson, 2005; Stelzer et al., 2011; Zelazo, 2003). Y el desarrollo del lenguaje permitirá la consolidación de procesos como la inhibición, el autocontrol, la memoria de trabajo y la autorregulación, que, a su vez, facilitarán el desarrollo de la planificación en la solución de problemas (Bauselas, 2010).

Según Isquith et al. (2005), existen dos tendencias en el ámbito de las evaluación de las FE en preescolares. La primera, se centra en tareas de rendimiento estándar que se aplican directamente a los menores, mientras que la segunda busca medir mediante el reporte de padres y docentes los aspectos más globales de la autorregulación en el contexto cotidiano. Estos autores proponen un enfoque mixto de evaluación junto con una perspectiva ecológica mediante la que se mida el impacto funcional de las FE en la realización de tareas cotidianas a través de pruebas estandarizadas y validadas.

El Behavior Rating Inventory of Executive Functions - Preschool Version (BRIEF-P; Gioia et al., 2003) es la primera escala de calificación estandarizada diseñada para medir las manifestaciones conductuales de la función ejecutiva en niños(as) de edad preescolar, que ha evidenciado su utilidad en la práctica clínica y en la investigación, en estudios como el de Clark. Pritchard y Woodward (2010) en el que concluyeron que una medida temprana de FE como la BRIEF-P podría ser útil para identificar niños(as) con dificultades en la adquisición de conceptos y habilidades matemáticas; así como la investigación de Mahone y Hoffman (2007), quienes evaluaron las FE en 25 niños con trastorno por déficit de atención e hiperactividad (TDAH) y 25 niños del grupo de control, encontrando que el BRIEF-P era sensible a los síntomas de este trastorno.

Sin embargo, el BRIEF-P no ha sido todavía validado en países como Colombia, donde se requiere este tipo de instrumentos de evaluación, debido a la presencia de condiciones adversas para el sano desarrollo de los niños(as), como la desigualdad, el conflicto armado, los malos tratos infantiles y el consumo abusivo de bebidas alcohólicas, así como la alta prevalencia de trastornos como el TDAH (Ministerio de Salud de Colombia \& Colciencias, 2015), asociados a déficits en FE que requieren una evaluación a temprana edad.

Conforme a lo anterior, este estudio se planteó como objetivo general adaptar el cuestionario BRIEF-P y evaluar sus propiedades psicométricas en una muestra de niños y niñas colombianos 2 a 4 años y 11 meses de edad. Se formularon los siguientes objetivos específicos: a) realizar una adaptación del inglés al castellano del BRIEF-P y b) examinar sus propiedades psicométricas con diferentes pruebas estadísticas de confiabilidad y validez.

\section{Método}

\section{Tipo De Estudio}

El presente estudio de tipo instrumental, se enmarca dentro de las características de una investigación de enfoque empírico-analítico, de tipo descriptivo, transversal y de un solo grupo (Méndez \& Nahimira, 2000). Dado el tamaño de la muestra, se considera que éste es un estudio preliminar de la evaluación de las propiedades psicométricas del instrumento. 


\section{Participantes}

Se tomó como población el total de niños y niñas de 2 a 4 años y 11 meses de edad del área urbana y rural del Municipio de Tunja (Colombia), que asciende a 12.000 individuos aproximadamente, según el Programa de las Naciones Unidas para el Desarrollo y la Alcaldía Mayor de Tunja (2012). Se calculó el tamaño muestral con el programa STATS versión 2.0, con un intervalo de confianza de $95 \%$ y un error de $9 \%$, para un total de 118 individuos. De esta manera, se seleccionaron 125 preescolares de nueve instituciones educativas de la ciudad de Tunja, con el fin de superar el valor mínimo arrojado por dicho programa estadístico, previendo deserciones por parte de algunos participantes. Los niños y niñas seleccionados, 60 mujeres (48\%) y 65 varones (52\%), tenían entre 2 años 0 meses y 4 años 11 meses y una media de 51.67 meses de edad.

El 25.6\% de los participantes vivían en barrios de estrato socioecónómico bajo-bajo, el $36 \%$ en barrios de estrato bajo, el $30.4 \%$ en barrios de estrato medio-bajo y el $7.2 \%$ de estrato medio. El $70 \%$ de la muestra vivía en el área urbana de la ciudad. El $49.6 \%$ de la muestra pertenecía a una familia nuclear, el $8 \%$ a una familia monoparental, el $1.6 \%$ a una familia recompuesta, el $28 \%$ a una familia extensa y el $10.4 \%$ a una familia extensa-monoparental. El $11.2 \%$ de las 125 madres y padres de los niños y niñas que participaron en este estudio manifestó haber cursado primaria completa, el $36.8 \%$ bachillerato, el $11.2 \%$ estudios técnicos o tecnológicos, el $18.4 \%$ estudios universitarios y el $3.2 \%$ estudios de posgrado.

Según el reporte de los padres y la observación directa efectuada durante la evaluación, el $78.4 \%$ de los participantes eran diestros y el $7.2 \%$ zurdos, mientras que el $9.6 \%$ de la muestra, probablemente por su corta edad, no evidenciaba todavía una tendencia definida. El 75\% de las madres de los niños y niñas vinculados al estudio tenían entre 16 y 30 años de edad al momento del parto. El $81.6 \%$ de los participantes tuvieron un parto a término y el $86 \%$ presentaron partos normales, es decir, por vía vaginal sin necesidad de inducción o instrumentación, mientras que el $17.6 \%$ de los participantes tuvieron complicaciones durante el embarazo, el 12\% presentaron complicaciones durante el parto y el $13.6 \%$ a nivel posnatal. En estos casos se verificó que estas complicaciones no hubieran generado retrasos significativos en los hitos del desarrollo motor y cognitivo propios de los primeros años

\section{Instrumentos}

Behavior Rating Inventory of Executive Function - Preschool Version (BRIEF-P) (Gioia et al., 2003). Es el primer instrumento estandarizado diseñado específicamente para medir múltiples aspectos del funcionamiento ejecutivo en niños(as) de edad preescolar, según el reporte de su padre o madre, profesor o cuidador, a través de escalas de medición de la inhibición, el cambio, el control emocional, la memoria de trabajo y la planificación/organización. Es útil en la evaluación de niños(as) en edad preescolar con condiciones médicas, enfermedades neurológicas adquiridas y del desarrollo como la prematuridad, dificultades de aprendizaje, trastornos de atención, trastornos del lenguaje, lesiones cerebrales traumáticas, exposición al plomo y trastornos generalizados del desarrollo.

Consta de un formulario de valoración único con 63 ítems tipo Likert ("Nunca": 0, "Algunas veces": 1 y "Siempre": 2), que permite evaluar las FE del niño(a) en el contexto de su entorno cotidiano, tanto en la casa como en la escuela, mediante tres índices generales (Autocontrol Inhibitorio, Flexibilidad y Metacognición Emergente), un puntaje compuesto y dos escalas de validez (Inconsistencia y Negatividad). 
El índice de Autocontrol Inhibitorio, a su vez, es la suma de las puntuaciones en las siguientes escalas:

Inhibición (16 ítems). Referente al control de los impulsos y el comportamiento, examina si el niño(a) se detiene apropiadamente y modula la propia conducta en el momento adecuado en el contexto adecuado.

Cambio (10 ítems). Evalúa si el niño(a) se mueve libremente de una actividad o otra, o de un aspecto de un problema a otro, según la situación lo requiera, haciendo transiciones y resolviendo los problemas de forma flexible.

El índice de Flexibilidad, además de la escala de Cambio, incluye el puntaje obtenido en la siguiente escala:

Control emocional (10 ítems). Examina si el niño(a) modula la respuesta emocional de manera adecuada según la situación o contexto.

Por su parte, el índice de Metacognición Emergente abarca las siguientes escalas:

Memoria de trabajo (17 ítems). Evalúa el mantenimiento de la información en la mente con el fin de completar una tarea o producir una respuesta apropiada y si el niño(a) permanece en una actividad o producir una respuesta apropiada.

Planificación/Organización (10 ítems). Examina si el niño(a) anticipa los acontecimientos o las consecuencias futuras, utiliza metas e instrucciones para guiar su comportamiento según el contexto y desarrolla o implementa pasos apropiados para llevar a cabo una tarea o acción.

Las puntuaciones directas en las escalas se interpretan de modo que entre más alto el puntaje obtenido, menor la capacidad del individuo para tener un desempeño adecuado en esa función.

Sumado a lo anterior, el instrumento contiene las siguientes escalas de validez:

Inconsistencia (10 pares de ítems). Indica el grado en que el encuestado responde ítems similares en forma inconsistente.

Negatividad (10 ítems). Mide el grado en que el encuestado responde ítems seleccionados de una manera inusualmente negativa.

Para la construcción de los ítems los autores se basaron en entrevistas clínicas con padres y profesores sobre las descripciones y quejas más comunes sobre déficits en FE, así como en el concepto de neuropsicólogos y pediatras con experiencia en la población blanco. Los datos normativos ofrecidos por los autores se basan en las calificaciones de los niños(as) de 460 padres y 302 docentes de áreas urbanas, suburbanas y áreas rurales de Estados Unidos, con base en las estimaciones del Censo de 1999 sobre raza/etnia, género, nivel socioeconómico y edad. Las muestras clínicas incluyeron niños(as) con TDAH, prematuridad, trastornos del lenguaje, trastornos del espectro autista y un grupo clínico mixto. Se efectuaron pruebas de consistencia interna, estabilidad test-retest, acuerdo inter-escalas y validez de constructo. 
La consistencia interna, calculada mediante el alfa de Crombach, osciló entre .80 y .97, aunque el acuerdo ínter-escalas entre padres y maestros fue bajo (.19). La estabilidad test-retest se examinó con una submuestra de 52 padres, con un lapso de 4.5 semanas en promedio, oscilando entre .78 y .90, mientras que con una submuestra de 67 docentes, dicha estabilidad fluctuó entre .65 y .94, con un lapso promedio entre medidas de 4.2 semanas.

Los autores evaluaron la relación convergente y discriminante entre el BRIEF-P y otras medidas presuntamente relacionadas y no relacionadas, mediante la matriz multirasgos-multimétodos, a través de instrumentos como Inattention Scale of the ADHD Rating Scale IV - Preschool Version (ADHD-IV-P) (McGoey, Bradley-Klug, Crone. Shelton, \& Radcliffe, 2000), Child Behavior Checklist (CBCL) (Achenbach \& Rescorla, 2000) y Behavior Assessment System for Children (BASC) (Reynolds \& Kamphaus, 1992), encontrándo correlaciones significativas entre la escala de Memoria de Trabajo de la BRIEF-P y medidas de atención, y entre Inhibición y medidas de hiperactividad y agresión. Las escalas del BRIEF-P mostraron baja correlación con constructos no relacionados, como medidas de ansiedad y quejas somáticas.

Pruebas de ejecución. Con el fin de evaluar la validez de criterio del BRIEF-P, se aplicaron a los niños(as) las siguientes pruebas descritas por Carlson, (2005), utilizadas ampliamente en la investigación neuropsicológica infantil con niños(as) en edad preescolar, unas dirigidas para niños(as) de dos años y las otras para los niños(as) de tres años:

Oso/dragón. El evaluador presenta a los niños(as) un títere de oso amable (usando una voz suave y aguda) y un títere de dragón travieso (usando una voz gruesa); se les explica que en este juego ellos deben hacer lo que el oso les diga que hagan, por ejemplo, "toca tu nariz", pero no hacer lo que el dragón les diga. Luego de practicar, se realizan diez ejecuciones con el oso y el dragón alternando quien manda. Los niños(as) permanecen sentados en una mesa durante la tarea, en la que todas las acciones involucran movimientos de las manos. Las ejecuciones ante las ordenes dadas por el títere del dragón son tomadas como un índice de autocontrol, puntuándose cero (0) si el niño(a) se mueve y uno (1) si no lo hace, y puntuándose individualmente cada ensayo.

Categorización inversa. A los niños(as) de dos años se les presentan dos cubos y se les pide que ayuden al evaluador a ubicar bloques grandes en el cubo grande y bloques chiquitos en el cubo pequeño, mientras que a los de tres años se les pide que ayuden a ubicar los animales mamá en un cubo de mamás y los animales bebés en un cubo de bebés. Luego el evaluador sugiere que participen en un juego tonto en el que se reversa este esquema de categorización. Se realizan 12 ensayos, con un recordatorio de la regla en cada intento en el caso de los niños(as) de dos años y un recordatorio a la mitad de los ensayos, para el caso de los niños(as) de tres años. La puntuación es el número total de respuestas correctas.

Susurro. El evaluador le pregunta al niño(a) si puede susurrar su nombre, luego le presenta consecutivamente 10 tarjetas que representan 10 dibujos animados, seis familiares y cuatro poco familiares para la mayoría de preescolares. Se les pide que susurre el nombre de cada personaje, diciéndole que está bien que no los conozcan a todos. En cada ensayo recibe una puntuación de uno (1) si susurra el nombre del personaje y de cero (0) si usa una voz normal o grita dicho nombre, no se puntuá la respuesta "No sé". Los personajes poco familiares sirven para controlar la emoción que pueden experimentar los niños(as) al ver personajes muy conocidos. 
Contar y etiquetar. Al niño(a) se le muestran tres objetos: una llave, un zapato y un perro de juguete, y se le pide que los nombren. Luego el evaluador invita al niño(a) a contar los objetos, sin nombrarlos, mostrando cómo se hace asignándole un número a cada uno (por ejemplo, 1 es una llave, 2 es un zapato y 3 es un perro). Se realizan dos ensayos con diferentes objetos. A los niños(as) se les puntua de manera incorrecta si cuentan los objetos y luego los nombran o viceversa o si dicen "uno es una llave, uno es un zapato, uno es un perro" o algo similar.

Día/noche. El evaluador involucra a los niños(as) en una conversación acerca de cuando sale el sol (en el día) y cuando empieza a salir la luna (en la noche), luego presenta una tarjeta blanca con un sol amarillo dibujado en ella y una tarjeta negra con una luna blanca y estrellas. A los niños(as) se les instruye que en este juego tienen que decir "noche" para la carta del sol y "día" para la carta de la luna. Después de un pequeño entrenamiento se realizan 16 ensayos con cada carta presentada desde debajo de la mesa, en un orden fijo y pseudoaleatorio. No hay descanso ni recordatorio de reglas. Se registra el número de respuestas correctas en los 16 ensayos.

Retraso en el regalo -envoltorio-. El evaluador dice a los niños(as) que han hecho un gran trabajo en las actividades anteriores y que van a recibir un premio, y que se le olvidó envolver el regalo. Les pide a los niños(as) que se den vuelta para que él pueda envolverlo y les advierte que no pueden husmear. Luego, el evaluador envuelve una pequeña caja de regalo de manera ruidosa en una bolsa papel, por 60 segundos. Se califica incorrectamente si el niño presenta el comportamiento de husmear.

Retraso en el regalo -cinta- (niños-as- de dos años). El evaluador felicita a los niños(as) por hacer un buen trabajo y les anuncia un regalo (una gran bolsa de regalo con un regalo envuelto adentro), luego les dice que se le olvidó poner una cinta en el regalo y le pide a los niños(as) que esperen sin abrirlo hasta que vuelva con la cinta. El evaluador se va del cuarto por tres minutos, luego vuelve con la cinta e invita a los niños(as) a abrir el regalo (si no lo habían hecho ya). Se registra el comportamiento de los niños(as) durante la ausencia del evaluador frente a la demanda de no abrirlo.

Cambio de lugar (2 años). El evaluador presenta a los niños(as) varios cajones de madera. Tres de estos cajones tienen tiritas para abrirlas, con un símbolo pegado en cada una (por ejemplo, un círculo verde). Se les muestra a los niños(as) como jalar la tirita para abrir el cajón. Después de un pequeño entrenamiento, el evaluador plantea como premio un dulce dentro de uno de los cajones y les pide a los niños(as) que lo cojan. Después de que el niño(a) ha tomado el dulce del cajón correcto en tres ocasiones consecutivas en el mismo cajón, el evaluador visiblemente cambia el lugar de escondite del premio en un cajón diferente (con un símbolo diferente pegado) y deja pasar 10 segundos antes de invitar al niño(a) a coger el premio. Se califica incorrectamente si el niño(a) persevera buscando en el cajón anterior.

\section{Procedimiento}

1. Adaptación del instrumento BRIEF-P al castellano. Mediante el método de la doble traslación, en el que participaron dos personas bilingües certificadas mediante un examen estandarizado internacional: una de las cuales efectuó la traducción del instrumento al castellano y la otra revisó el instrumento traducido al castellano y lo tradujo nuevamente al idioma inglés. Luego la primera traductora comparó la 
Junto con el instrumento se aplicó una ficha de recolección de datos y antecedentes clínicos para descartar niños(as) participantes con déficits del desarrollo, trastornos generalizados de desarrollo o condiciones médicas que pudieran afectar su desempeño. versión original con la traducida del castellano al inglés para determinar las posibles inconsistencias y efectuar las correcciones del caso teniendo en consideración factores lingüísticos y culturales.

2. Pilotaje y juicio de expertos. Se hizo una aplicación preliminar del instrumento traducido, según lo explicado anteriormente, a un grupo de 20 padres y madres, con características de inclusión idénticas a las descritas para la muestra, con el fin de verificar la comprensión de los ítems y hacer ajustes a las características idiomáticas del instrumento. En este proceso surgieron cuestiones relacionadas con la redacción de algunos ítems y la escala de calificación. Después de estos ajustes el documento se sometió a juicio de expertos, en el cual participaron cuatro psicólogos con maestría o doctorado en neuropsicología o neurociencias y experiencia docente e investigativa en neuropsicología infantil.

3. Aprobación de la versión por parte de los autores de la prueba. El documento resultante fue remitido por la empresa distribuidora oficial del instrumento para su revisión (PAR Instruments Corporation) a uno de los autores de la prueba (Peter Isquith) y tras algunas correcciones sugeridas por dicho autor, fue aprobada por dicha empresa.

4. Administración del instrumento. Se citó a los padres a un auditorio con condiciones de iluminación y temperatura adecuadas, en grupos de máximo 20 personas, en su mayoría en horas de la mañana, con el fin de garantizar condiciones óptimas de atención. El aplicador resolvió las inquietudes del caso durante el diligenciamiento del cuestionario, el cual fue contestado por 96 madres, 9 padres y 20 por ambos miembros de la pareja, para un total de 125. A los dos padres que habían cursado primaria incompleta y a todos aquellos con mayor escolaridad que mostraron dificultades de comprensión de los ítems, se les asesoró e incluso se les leyó la totalidad de la prueba en los casos que fue necesario.

Junto con el instrumento se aplicó una ficha de recolección de datos y antecedentes clínicos para descartar niños(as) participantes con déficits del desarrollo, trastornos generalizados de desarrollo o condiciones médicas que pudieran afectar su desempeño. Una vez firmado el consentimiento informado por parte de los padres, se aplicó a los niños y niñas las tareas de evaluación de FE que sirvieron para evaluar la validez de criterio del instrumento. Cada una de estas pruebas duró en promedio cinco minutos, por lo que esta evaluación duró entre 20 y 30 minutos.

Con el fin de calcular la estabilidad test-retest, se realizó una segunda aplicación del instrumento a un subgrupo de 15 padres y madres de familia, tras un lapso de entre cuatro y ocho semanas (seis semanas en promedio).

5. Análisis estadístico e informe. Los resultados se incorporaron en el programa estadístico SPSS versión 22 y se efectuaron los mismos análisis de confiabilidad implementados en la validación original (Gioia et al., 2003): a) Alfa de crombach, con el fin de examinar la consistencia interna y b) estabilidad test-retest mediante la prueba de correlación de Pearson.

Para evaluar la validez de criterio se estimó la correlación entre las pruebas de ejecución de Carlson, 2005 y las escalas de BRIEF-P que miden las mismas funciones, a saber: a) Oso/dragón: Inhibición; b) categorización inversa: Cambio, Planificación/ Organización; c) susurro: Inhibición, Control Emocional; d) Contar y Etiquetar: Memo- 
ria de Trabajo, Planificación/Organización; e) día/noche: Inhibición; f) retraso en el regalo: Control Emocional y g) cambio de lugar: Cambio. Para ello se utilizó la prueba de correlación de Pearson, mientras que para evaluar la validez de constructo, esto es, examinar si la estructura factorial era equivalente a los resultados de la validación original, se utilizó el análisis factorial confirmatorio con rotación varimax.

\section{Consideraciones éticas}

Para el presente trabajo se tuvieron en cuenta las consideraciones éticas consagradas en la Ley 1090 de 2006 (por la cual se reglamenta el ejercicio de la profesión de Psicología en Colombia), con respecto al uso de material psicotécnico, la investigación científica y la propiedad intelectual, así como los principios referentes a la salvaguarda de la dignidad, el respeto, el bienestar y la confidencialidad de los participantes, conforme a los cuales los padres firmaron el consentimiento informado para la utilización de los resultados de la investigación, dado que se trata de menores de edad. A la luz de la Resolución 8430 de 1993 del Ministerio de Salud de Colombia, este estudio se clasifica como de riesgo mínimo, dado que se aplicaron pruebas psicométricas sin manipular la conducta de los sujetos. Previa autorización de las instituciones educativas, se implementó un formato de consentimiento informado para los padres que contiene la información estipulada por dicha norma y se buscó el asentimiento de los niños(as) explicándoles de manera sencilla las diferentes pruebas de ejecución.

\section{Resultados}

En la tabla 1 se presentan los datos de la media y desviación estándar obtenida en cada una de las escalas e índices de la prueba, a nivel test y retest, así como las correlaciones que se presentaron entre ambas mediciones y los índices alfa de crombach. Los resultados señalan altos grados de estabilidad, con correlaciones altamente significativas entre las mediciones test y retest y valores que oscilaron entre .566 y .790. Los índices alfa de crombach, por su parte, oscilaron entre .697 y .887, con un alfa general de .939 , resultados que indican una alta consistencia interna del instrumento.

Tabla 1. Estabilidad test-retest y consistencia interna de las escalas e índices de la prueba

\begin{tabular}{|c|c|c|c|c|c|c|c|}
\hline \multirow{2}{*}{ Escala } & \multicolumn{2}{|c|}{ Test } & \multicolumn{2}{|c|}{ Retest } & \multirow{2}{*}{$\begin{array}{l}\text { Correlación } \\
\text { de Pearson }\end{array}$} & \multirow{2}{*}{$\begin{array}{c}\text { Significación } \\
\text { (bilateral) }\end{array}$} & \multirow{2}{*}{$\begin{array}{c}\text { Alfa de } \\
\text { Crombach }\end{array}$} \\
\hline & $x$ & $D X$ & $x$ & $D X$ & & & \\
\hline Inhibición & 27.71 & 5.473 & 27.13 & 5.502 & $.647^{* *}$ & .009 & .831 \\
\hline Cambio & 14.40 & 3.317 & 14.00 & 2.619 & $.790^{* *}$ & .000 & .761 \\
\hline Control Emocional & 17.60 & 4.105 & 17.60 & 3.439 & $.685^{* *}$ & .005 & .822 \\
\hline Memoria de Trabajo & 26.88 & 5.629 & 25.80 & 5.031 & $.566^{*}$ & .028 & .859 \\
\hline Planeación/ Organización & 16.70 & 3.251 & 16.27 & 3.081 & $.571^{*}$ & .026 & .697 \\
\hline Autocontrol & 42.11 & 7.916 & 41.13 & 6.770 & $.686^{* *}$ & .005 & .865 \\
\hline Flexibilidad & 31.98 & 6.573 & 31.60 & 5.767 & $.754^{* *}$ & .001 & .862 \\
\hline Metacogn. Emergente & 43.58 & 8.287 & 42.07 & 7.704 & $.580^{*}$ & .023 & .887 \\
\hline Global Compuesta & 102.49 & 19.773 & 94.13 & 27.404 & $.688^{* *}$ & .005 & .939 \\
\hline
\end{tabular}

Nota. X: Media; DX: Desviación estándar. ${ }^{*} p \leq .05{ }^{* *} p \leq .01$

Por otra parte, los resultados obtenidos en la medida de adecuación de muestreo Kaiser-Meyer-Olkin (.822) y la prueba de esfericidad de Bartlett $(327,330)$, con 10 grados de libertad y significancia superior a 0.001, indican que era apropiado efectuar un análisis factorial para este instrumento, el cual, mediante el método de extracción de análisis de componentes principales, evidenció cinco factores que explicaban el $100 \%$ de la varianza, uno de los cuales explicaba el $67.5 \%$ de la misma, como se evidencia en la tabla 2. 
Tabla 2. Varianza total explicada

\begin{tabular}{|c|c|c|c|c|c|c|}
\hline \multirow[b]{2}{*}{ Componente } & \multicolumn{3}{|c|}{ Autovalores iniciales } & \multicolumn{3}{|c|}{$\begin{array}{c}\text { Sumas de extracción de cargas al } \\
\text { cuadrado }\end{array}$} \\
\hline & Total & $\% \sigma^{2}$ & $\%$ acum. & Total & $\% \sigma^{2}$ & $\%$ acum. \\
\hline 1 & 3.375 & 67.499 & 67.499 & \multirow{5}{*}{3.75} & \multirow{5}{*}{67.499} & \multirow{5}{*}{67.499} \\
\hline 2 & .657 & 13.139 & 80.639 & & & \\
\hline 3 & .440 & 8.802 & 89.441 & & & \\
\hline 4 & .284 & 5.683 & 95.124 & & & \\
\hline 5 & .244 & 4.876 & 100.000 & & & \\
\hline
\end{tabular}

El análisis factorial de componentes principales con rotación varimax evidencia una alta correlación entre todas las escalas del instrumento; información que se presenta en la tabla 3. La varianza obtenida en cada una de las escalas en la matriz de componentes fue la siguiente: a) Inhibición: .869, b) Cambio: .807, c) Control Emocional: .770, d) Memoria de Trabajo: .825 y e) Planificación/Organización: .834.

Tabla 3. Correlación entre escalas

\begin{tabular}{lccccc}
\hline \multicolumn{1}{c}{ Escala } & Inhibición & Cambio & Control Emocional & Memoria de Trabajo & Planeación \\
\hline Inhibición & 1.000 & $.598^{* * *}$ & $.694^{* * *}$ & $.605^{* * *}$ & $.655^{* * *}$ \\
Cambio & $.598^{* * *}$ & 1.000 & $.568^{* * *}$ & $.594^{* * *}$ & $.564^{* * *}$ \\
Control Emocional & $.694^{* * *}$ & $.568^{* * *}$ & 1.000 & $.456^{* * *}$ & $.469^{* * *}$ \\
Memoria de Trabajo & $.605^{* * *}$ & $.594^{* * *}$ & $.456^{* * *}$ & 1.000 & $.722^{* * *}$ \\
Planeación & $.655^{* * *}$ & $.564^{* * *}$ & $.469^{* * *}$ & $.722^{* * *}$ & 1.000 \\
\hline
\end{tabular}

${ }^{* * *} \mathrm{p} \leq .001$

Teniendo en cuenta los resultados obtenidos mediante el análisis factorial, se extrajeron las cargas factoriales de cada uno de los ítems del instrumento para un máximo de cinco factores (véase la Tabla 4).

Tabla 4. Correlación de Pearson entre las escalas del BRIEF-P y las pruebas de desempeño.

\begin{tabular}{ccccccccc}
\hline \multicolumn{2}{c}{ Escalas } & $\begin{array}{c}\text { Oso/ } \\
\text { Dragón }\end{array}$ & $\begin{array}{c}\text { Categ. } \\
\text { Inversa }\end{array}$ & Susurro & $\begin{array}{c}\text { Contar y } \\
\text { Etiquetar }\end{array}$ & $\begin{array}{c}\text { Día/ } \\
\text { Noche }\end{array}$ & $\begin{array}{c}\text { Retraso en } \\
\text { el Regalo }\end{array}$ & $\begin{array}{c}\text { Cambio de } \\
\text { Lugar }\end{array}$ \\
\hline \multirow{2}{*}{ Inhibición } & Correlac. & $-.258^{* *}$ & -.167 & $-.239^{* *}$ & $-.176^{*}$ & $-.200^{*}$ & -.094 & -.169 \\
& Sig. (bilat.) & .004 & .063 & .007 & .050 & .025 & .299 & .059 \\
\hline \multirow{2}{*}{ Cambio } & Correlac. & -.165 & $-.199^{*}$ & -.147 & -.098 & -.119 & .063 & -.049 \\
& Sig. (bilat.) & .066 & .026 & .103 & .277 & .185 & .489 & .584 \\
\hline Control & Correlac. & $-.243^{* *}$ & -.069 & -.144 & -.111 & $-.243^{* *}$ & -.095 & $-.311^{* *}$ \\
Emocional & Sig. (bilat.) & .006 & .446 & .108 & .217 & .006 & .294 & .000 \\
\hline Memoria de & Correlac. & -.174 & -.135 & -.109 & $-.192^{*}$ & -.174 & -.026 & $-.201^{*}$ \\
Trabajo & Sig. (bilat.) & .053 & .134 & .227 & .032 & .053 & .774 & .024 \\
\hline \multirow{2}{*}{ Planeación } & Correlac. & -.132 & -.021 & -.126 & -.172 & -.080 & .051 & -.152 \\
& Sig. (bilat.) & .141 & .819 & .163 & .056 & .373 & .571 & .091 \\
\hline Autocontrol & Correlac. & $-.247^{* *}$ & $-.198^{*}$ & $-.227^{*}$ & -.163 & $-.188^{*}$ & -.038 & -.138 \\
Inhibitorio & Sig. (bilat.) & .005 & .027 & .011 & .070 & .036 & .670 & .125 \\
\hline \multirow{2}{*}{ Flexibilidad } & Correlac. & $-.231^{* *}$ & -.140 & -.161 & -.116 & $-.215^{*}$ & -.023 & $-.221^{*}$ \\
& Sig. (bilat.) & .009 & .119 & .073 & .199 & .016 & .799 & .013 \\
\hline Metacogn. & Correlac. & -.170 & -.100 & -.123 & $-.198^{*}$ & -.150 & .002 & $-.196^{*}$ \\
Emergente & Sig. (bilat.) & .058 & .269 & .171 & .027 & .096 & .979 & .028 \\
\hline
\end{tabular}


Los resultados obtenidos con las pruebas de correlación entre las escalas del BRIEF-P y las pruebas de desempeño se muestran en la tabla 4 , los cuales evidencian correlaciones estadísticamente significativas entre: a) la escala de Inhibición y las pruebas de oso/dragón, susurro, contar y etiquetar y día/noche; b) la escala de Cambio con la prueba de categorización inversa; c) la escala de Control Emocional con las pruebas de oso/dragón, día/noche y cambio de lugar; d) la escala de Memoria de Trabajo y el índice de Metacognición Emergente, con las pruebas de contar y etiquetar y cambio de lugar. Asimismo, e) el índice de Autocontrol Inhibitorio correlacionó significativamente con las pruebas de oso/dragón, categorización inversa susurro y día/noche, y f) el índice de Flexibilidad con las pruebas de oso/dragón, día/ noche y cambio de lugar. La escala de Planificación/Organización no correlacionó significativamente con ninguna de las pruebas aplicadas.

\section{Discusión}

El objetivo de este trabajo fue adaptar y evaluar las propiedades psicométricas del BRIEF-P (Gioia et al., 2003), para lo cual se contó con una muestra de 125 niños y niñas en edad preescolar. Los resultados de análisis de consistencia interna del instrumento fueron favorables, con índices superiores a .80 en todos los índices, en la puntuación global compuesta y en tres de las cinco escalas del instrumento, siendo igual o superior a .70 en las dos escalas restantes. Estos valores son similares a los obtenidos en el estudio de validación original en el cual se hallaron índices que oscilaban entre .80 y .97 (Giogia et al., 2003) y confirman que los ítems del BRIEF-P presentan una buena consistencia interna y, por lo tanto, que el instrumento es preciso, y presenta bajos niveles de distorsión de medida (Gregory, 2012; Kerlinger \& Lee, 2002). Por otra parte, los resultados de las pruebas de correlación test-retest confirmaron la buena estabilidad del instrumento, con índices que oscilaron entre .57 y .79, a pesar del tamaño de la submuestra compuesta por 15 participantes a quienes se les aplicó dos veces el instrumento y el tiempo trascurrido entre dichas aplicaciones fue de seis semanas en promedio. En el estudio original, dichos valores oscilaron entre y .78 y .90, y el período entre las dos administraciones fue de cuatro semanas y media en promedio.

Con respecto a la validez de constructo, los resultados del análisis factorial confirmaron que hay cinco factores que pueden explicar el 100\% de la varianza, cantidad de factores que coincide con el número de escalas que tiene el instrumento, lo que señala que no es posible reducir dicho número de escalas. El análisis factorial también mostró que un factor explicaba el $67.5 \%$ de la varianza, lo que confirma que el instrumento mide un mismo constructo, en este caso, las FE en preescolares. Asimismo, las escalas correlacionaron entre sí con un alto nivel de significancia estadística ( $p \leq .001$ ) y con índices superiores a .50 en casi todos los casos, excepto en dos correlaciones en las cuales los valores fueron superiores a 40 .

En cuanto a la validez de criterio, las correlaciones observadas entre las escalas del BRIEF-P y las pruebas de ejecución descritas por Carlson, 2005 aplicadas a los niños(as) evidencian, en general, que el instrumento podría medir el comportamiento de los niños(as) asociado a las FE, pues, la escala de Inhibición correlacionó significativamente con los puntajes obtenidos en las pruebas de oso/dragón, susurro, contar y etiquetar y día/noche, mientras que la escala de Cambio correlacionó con la puntuación alcanzada en la prueba de categorización inversa y la de Memoria de Trabajo con los puntajes obtenidos con las pruebas de contar y etiquetar y cambio de lugar. No obstante, la escala de Control Emocional no correlacionó significativamente con pruebas como la de susurro y la de retraso en el regalo, sino con las pruebas de oso/dragón, día/noche y cambio de lugar, lo que indica que el control 
Pág 59

En conclusión, las diferentes pruebas estadísticas empleadas para evaluar las propiedades psicométricas del BRIEF-P, indican que la versión traducida y adaptada al castellano del mismo en la presente investigación es una medida válida y confiable para evaluar el desempeño en FE de niños y niñas colombianos en edad preescolar, si bien es conveniente contar con baremos provenientes de una muestra representativa de la población. emocional es una FE que se desarrolla posteriormente conforme a un incremento en el desarrollo cognitivo y social (Palacios \& Hidalgo, 1999). Ninguna de estas pruebas de ejecución correlacionó con la escala de Planeación/Organización, si bien podría ocurrir que estas no sean sensibles a esta FE y sea necesario implementar otras pruebas para ponderar la validez de criterio de dicha escala. El hecho de que las correlaciones obtenidas no fueron muy altas, por otra parte, ratifica la necesidad de utilizar pruebas de desempeño, observaciones y reportes de padres y profesores, para confirmar los resultados obtenidos con el instrumento.

En conclusión, las diferentes pruebas estadísticas empleadas para evaluar las propiedades psicométricas del BRIEF-P, indican que la versión traducida y adaptada al castellano del mismo en la presente investigación es una medida válida y confiable para evaluar el desempeño en FE de niños y niñas colombianos en edad preescolar, si bien es conveniente contar con baremos provenientes de una muestra representativa de la población. No obstante, se recomienda que el resultado obtenido en el cuestionario sea confrontado con pruebas de desempeño, reportes de informantes claves como docentes y padres, y observaciones clínicas para realizar una valoración válida y completa de las FE del niño o niña.

Esta investigación tuvo como principal fortaleza la posibilidad de implementar las mismas pruebas estadísticas para evaluar la confiabilidad y la validez del estudio original del BRIEF-P, lo cual permitió confirmar los resultados obtenidos en el mismo. Además, en la presente investigación se utilizaron pruebas de desempeño para ponderar la validez de criterio de la prueba, lo cual le dio un valor agregado con respecto a la valoración de las propiedades psicométricas del BRIEF-P. Los resultados obtenidos evidencian la conveniencia de utilizar la versión adaptada al castellano del instrumento, para su uso a nivel clínico y en investigación en el campo de la neuropsicología infantil en Colombia.

En cuanto a las limitaciones del estudio, cabe mencionar el tamaño de la muestra utilizada que le da un carácter de estudio preliminar, por lo cual es necesario adelantar estudios de baremación con muestras mayores, en los que al menos se cuente con 10 participantes por ítem del instrumento, conforme a lo recomendado por Kline (2005). Además, es conveniente utilizar instrumentos similares a los del estudio de validación original que no fueron utilizados en esta investigación, como el Behavioral Assessment System for Children (BASC) (Reynolds \& Kamphaus, 1992), para evaluar la validez discriminante del instrumento examinando la relación entre las FE y problemas de comportamiento internalizante y externalizante, así como instrumentos que examinen constructos relacionados con las FE para examinar la validez convergente del BRIEF-P. Asimismo, se recomienda examinar las diferencias por sexo en cada una de las escalas e índices del instrumento, y en los dos rangos de edad comparados por los autores: de 2 años 0 meses a 3 años 11 meses y de 4 años 0 meses a 5 años 11 meses (Gioia et al., 2003).

\section{Referencias}

Achenbach, T. M., \& Rescorla, L. A. (2000). Manual for the ASEBA Preschool Forms \& Profiles. Burlington, VT: University of Vermont, Research Center for Children, Youth, \& Families. http://store.aseba.org/MANUAL-FOR-ASEBA-PRESCHOOL-FORMS-PROFILE/productinfo/605/

Azcoaga, J. (2008). Estado de la neuropsicología infantil. Acta Neurológica Colombiana, 24(2), S5-S16. http://www.acnweb.org/acta/2008 24_S2 5.pdf 
Banco Mundial. (2011). Aprendizaje para todos: Invertir en los conocimientos y las capacidades de las personas para fomentar el desarrollo. Washington D.C.: Autor. http://siteresources.worldbank.org/EDUCATION/Resources/ESSU/4632921306181142935/Spanish Exec Summary 2020 FINAL.pdf

Bauselas, E. (2010). Función ejecutiva y desarrollo en la etapa preescolar. Boletín de Pediatría, 50(214), 272 - 276. http://sccalp.org/documents/0000/1674/BolPediatr2010_50_272-276.pdf

Carlson, S. (2005). Developmentally sensitive measures of executive function in preschool children. Developmental Neuropsychology, 28(2), 595-616. https://www. ncbi.nlm.nih.gov/pubmed/16144429

Clark, C., Pritchard, V., \& Woodward, L., (2010). Preschool executive functioning abilities predict early mathematics achievement. Developmental Psychology, 46(5), 1176-1191. https://www.ncbi.nlm.nih.gov/pubmed/20822231

Espy, K., Kaufmann, P., Glisky, M., \& McDiarmid, M. (2001). New procedures to assess executive functions in preschool children. The Clinical Neurospsychologist, 15(1), 46-58. https://www.ncbi.nlm.nih.gov/pubmed/11778578

Fletcher, J. (1996). Executive functions in children: Introduction to the special series. Developmental Neuropsychology, 12(1), 1-4. http://www.tandfonline.com/doi/ abs/10.1080/87565649609540636

Flores, J., Ostrosky-Solís, F., \& Lozano, A. (2008). Batería de Funciones Frontales y Ejecutivas: Presentación. Revista Neuropsicología, Neuropsiquiatría y Neurociencias, 8(1), 141-158. file:///C:/Users/salas/Downloads/Dialnet-BateriaDeFuncionesFrontalesYEjecutivasPresentacion-3987630.pdf

Gioia, G., Espy, K., \& Isquit, P. (2003). BRIEF-P: Behavior Rating Inventory of Executive Function Preschool Version. Professional Manual. Florida: PAR. http://www4. parinc.com/Products/Product.aspx?ProductID=BRIEF-P

González, M., \& Ostrosky, F. (2012). Estructura de las funciones ejecutivas en la edad preescolar. Acta de Investigación Psicológica, 2(1), 509-520. http://www.scielo. org.mx/scielo.php?script=sci arttext\&pid=S2007-48322012000100002

Gregory, R. (2012). Pruebas Psicológicas: Historia, principios y aplicaciones (6 ${ }^{\mathrm{a}}$ Ed.) México: Pearson. https://es.scribd.com/doc/253624241/Pruebas-Psicologicas-Historia-Principios-y-Aplicaciones

Isquith, P., Crawford, J., Espy, K., \& Gioia, G. (2005) Assessment of executive function in preschool-aged children. Mental Retardation \& Developmental Disabilities Research Reviews,11(3), 209-215. https://www.ncbi.nlm.nih.gov/pmc/articles/ PMC3648805/

Kerlinger, F., \& Lee H. (2002). Investigación del comportamiento (4⿻ Ed.) México: McGraw Hill. https://derechofunlam.files.wordpress.com/2015/09/investigacion del comportamiento - kerlinger fred n.pdf

Kline, R. B. (2005). Principles and practice of structural equation modeling (2a Ed.). New York, NY: Guilford Press. https://www.amazon.es/Principles-Practice-Structural-Equation-Methodology/dp/1606238760

McGoey, K.E., Bradley-Klug, K., Crone, D., Shelton, T.L., \& Radcliffe, J. (2000, Abril). Normative data of the ADHD-Rating Scale-IV-Preschool version. Trabajo presentado en la convencion annual de la National Association of School Psychologists, New Orleans, Louisiana. https://www.researchgate.net/publication/227265263 Parent and Teacher Ratings of Attention-DeficitHyperactivity_Disorder_in_Preschool_The_ADHD_Rating_Scale-IV_Preschool_Version

Mahone, E., \& Hoffman, J. (2007). Behavior ratings of executive function among preschoolers with ADHD. Clinical Neuropsychologist, 21(4), 569-586. https://www. ncbi.nlm.nih.gov/pubmed/17613979 
Méndez, I., \& Nahimira G. D. (2000). El protocolo de investigación: Lineamientos para su elaboración y análisis. México: Trillas. https://www.researchgate.net/publication/44510330 El Protocolo de investigacion lineamientos para su elaboracion y analisis Ignacio Mendez Ramirez et al

Ministerio de Salud de Colombia \& Colciencias. (2015). Encuesta Nacional de Salud Mental 2015 (Tomo 1). Bogotá: Autores. http://www.visiondiweb.com/insight/ lecturas/Encuesta_Nacional_de Salud_Mental_Tomo_I.pdf

National Scientific Council on the Developing Child (2007). The timing and quality of early experiences combine to shape brain architecture [documento de trabajo cinco]. Disponible en: http://www.developingchild.net

Oates, J., Karmiloff-Smith, A., \& Johnson, M. (2012). El cerebro en desarrollo. Aberystwyth: Cambrian Printers. https://bernardvanleer.org/app/uploads/2016/03/ El-cerebro-en-desarrollo-0131.pdf

Palacios, J. E, \& Hidalgo, V. (1999). Desarrollo de la personalidad desde los 6 años hasta la adolescencia. En J. E. Palacios, A. Marchesi, A. y C. Coll (Eds), Desarrollo psicológico y educación. Vol. 1: Psicología evolutiva (pp. 355-376). Alianza: Madrid. https://www.casadellibro.com/libro-desarrollo-psicologico-y-educacion-vol-1-psicologia-evolutiva/9788420686844/671310

Pérez, E., \& Capilla, A. (2008). Neuropsicología infantil. En J. Tirapu, M. Rios y F. Maestrú (Eds.), Manual de Neuropsicología (pp. 441-469). Barcelona: Viguera. https:// dialnet.unirioja.es/servlet/libro?codigo $=436709$

Programa de las Naciones Unidas para el Desarrollo, \& Alcaldía Mayor de Tunja. (2012). Política pública de primera infancia del Municipio de Tunja: Políticas Públicas para el logro de los ODM. Tunja: Linca Publicidad. http://186.116.13.48/ obsocial/documentos/PP\%20Primera\%20infancia_Tunja_2013.pdf

Reynolds, C. R., \& Kamphaus, R. W. (1992). Behavior assessment system for children. Circle Pines, Minnesota: American Guidance Service. http://www.pearsonclinical.com/education/products/100000658/behavior-assessment-system-for-children-second-edition-basc-2.html

Roselli, M., Jurado, M., \& Matute, E. (2008). Las funciones ejecutivas a través de la vida. Revista Neuropsicología, Neuropsiquiatría y Neurociencias, 8(1), 23-46. https:// www.google.com.co/url?sa $=t \& r c t=j \& q=\& e s r c=s \&$ source $=$ web \&cd $=1 \& v e d=0 a-$ hUKEwiZgeSK47 TAhXFMyYKHXH DiOQFggiMAA\&url=https\%3A\%2F\%2Fdialnet.unirioja.es\%2Fdescarga\%2Farticulo\%2F3987451.pdf\&usg=AFQjCNHV gJjlXgG1e6-PgofP30bRQaiAw

Samango-Sprouse, C. (2007). Frontal lobe development in childhood. En B. Miller \& J. Cummings (Eds,.), The human frontal lobes, functions and disorders (2a Ed., pp.576-596) New York: The Guilford Press. https://www.amazon.com/Human-Frontal-Lobes-Second-Neuropsychology/dp/1593853297

Sánchez-Carpintero, R., \& Narbona, J. (2004). El sistema ejecutivo y las lesiones frontales en el niño. Revista de Neurología, 39(2), 188-191. https://pdfs.semanticscholar.org/07d1/43a048f526dbd69b34f4370997590511d105.pdf

Stelzer, F., Cervigni, M., \& Martino, P. (2011). Desarrollo de las funciones ejecutivas en niños preescolares: una revisión de algunos de sus factores moduladores. Liberabit, 17(1), 93-100. http://www.scielo.org.pe/scielo.php?pid=S1729-48272 $011000100011 \&$ script $=$ sci arttext

Tirapu, U., \& Luna-Lario, P. (2008). Neuropsicología de las funciones ejecutivas. En J. Tirapu, M. Rios \& F. Maestrú (Eds.), Manual de neuropsicología (p. 221 - 256). Barcelona: Viguera. http://www.viguera.com/es/libros/25-manual-de-neuropsicologia-2-ed.html 
Thorella, L., \& Wåhlstedt, C., (2006). Executive functioning deficits in relation to symptoms of ADHD and/or ODD in preschool children. Infant and Child Development, 15, 503-518. http://onlinelibrary.wiley.com/doi/10.1002/icd.475/pdf

Trujillo, N., \& Pineda, D. (2008). Función ejecutiva en la investigación de los trastornos del comportamiento del niño y del adolescente. Revista Neuropsicología, Neuropsiquiatría y Neurociencias, 8(1), 77-94. https://dialnet.unirioja.es/servlet/articulo? codigo $=3987502$

Walker, S. P., Wachs, T. D., Grantham-McGregor, S., Black, M. M., Nelson, C. A., Huffman, S.... Richter, L. (2011). Inequality in early childhood: risk and protective factors for early child development. The Lancet, 378, 1325-1338. doi: http://dx.doi. org/10.1016/S0140-6736(11)60555-2

Zelazo, P. (2003). The development of executive function. Monographs of the Society for Research in Child Development, 68(3), 1-27. https://www.ncbi.nlm.nih.gov/ pubmed/14723273 